\title{
Structural Organization of Tropism-Switching Bordetella Bacteriophage Revealed by Integrative Electron Cryotomography and Cryomicroscopy
}

\author{
Wei Dai***, Asher Hodes***, Wong H. Hui*, Peng Ge***, Ivo Atanasov*, Jeff F. Miller*******, \\ Z. Hong Zhou*** \\ * Department of Pathology and Laboratory Medicine, University of Texas Medical School at \\ Houston, Houston, Texas 77030; \\ ** Program in Structural and Computational Biology and Molecular Biophysics, Baylor College of \\ Medicine, Houston, TX 77030; \\ *** Department of Microbiology, Immunology and Molecular Genetics, David Geffen School of \\ Medicine, University of California, Los Angeles, CA 90095 \\ **** The Molecular Biology Institute, University of California, Los Angeles, CA 90095
}

Tropism-switching Bordetella bacteriophages provide a model system for the studies of hostrange evolution. These phages have the ability to rapidly switch infection specificity by site-directed sequence transfer from a donor template repeat to a recipient variable repeat that encodes the $\mathrm{C}$ terminus of phage protein Mtd (major tropism determinant) [1, 2]. Mtd variation enables tropism switching by altering Mtd's specificity for receptors on the bacterial surface. Although the crystal structure of Mtd proteins had recently been determined, there has been little structural information on the overall organization of the phage and on its interaction with host bacteria [3]. To fill this gap, we have determined a multi-scale three-dimensional (3D) model of the Bordetella bacteriophage, BPP-1, revealing the structures of the head, the base plate, and the tail fibers, using an integrative approach of electron cryotomography (cryoET) and electron cryomicroscopy (cryoEM) (Fig. 1) [4]. A subnanometer-resolution $(9 \AA)$ structure of the head was obtained by icosahedral averaging 13,000 cryoEM particle images collected on a 16 megapixel CCD camera at 59k magnification. The head is consisted of 12 pentons, and 360 capsid subunits on a $\mathrm{T}=7$ icosahedral lattice. This map reveals the secondary structural elements in the capsid protein subunit. A long helix is identified close to the three-fold axis, which likely contributes significantly to the integrity and stability of the capsid shell. An 18-Á medium resolution structure was obtained by averaging over 30,000 particle images without imposing any symmetry to resolve the tail assembly. The tail assembly is also called the base plate that is composed of a center cylinder and 6 surrounding leaflets. The structural details of the transition from the underlying 5 fold symmetric subunit to the quasi 6 fold symmetry of the leaflets are determined (Fig. 1h-j). In addition, the packing pattern of the DNA inside the head is clearly revealed. Finally, a cryoET reconstruction was obtained to establish a low resolution model of the whole particle, including the extended, flexible tail fibers. 3D averaging of the tail fibers revealed a two-lobe structure at the distal end that propels clockwise from the bottom view, suggesting a possible mechanism of phage motion, host interactions and cooperativities. Taken in its entirety, this first model of the BPP-1 phage provides valuable structural clues into the extraordinary adaptability, variability and cooperativity of this phage strain.

\section{References}

[1] M. Liu et al., Science, 295 (2002) 2091-2094.

[2] S. Doulatov et al., Nature, 431 (2004) 476-481.

[3] S. A. McMahon et al., Nat Struct Mol Biol., 10 (2005) 886-892.

[4] Y. Liang et al., J. Struct. Biol., 137 (2002) 292-304. 

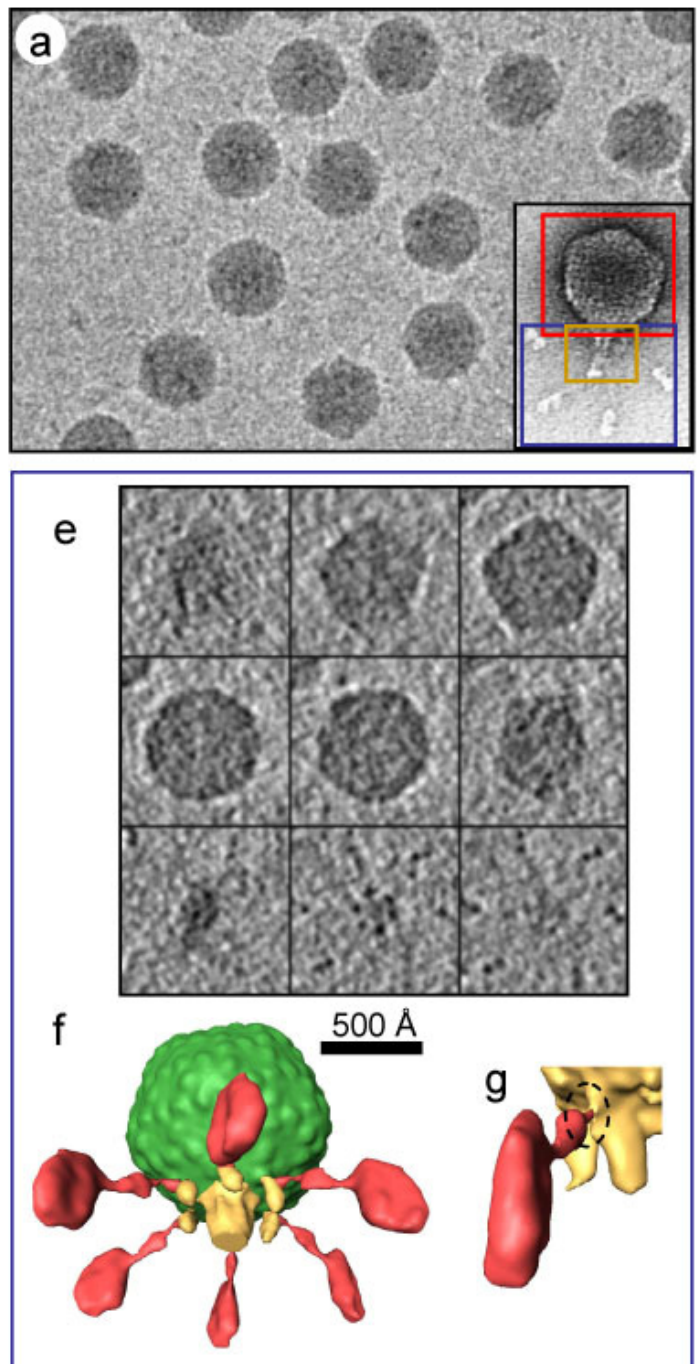
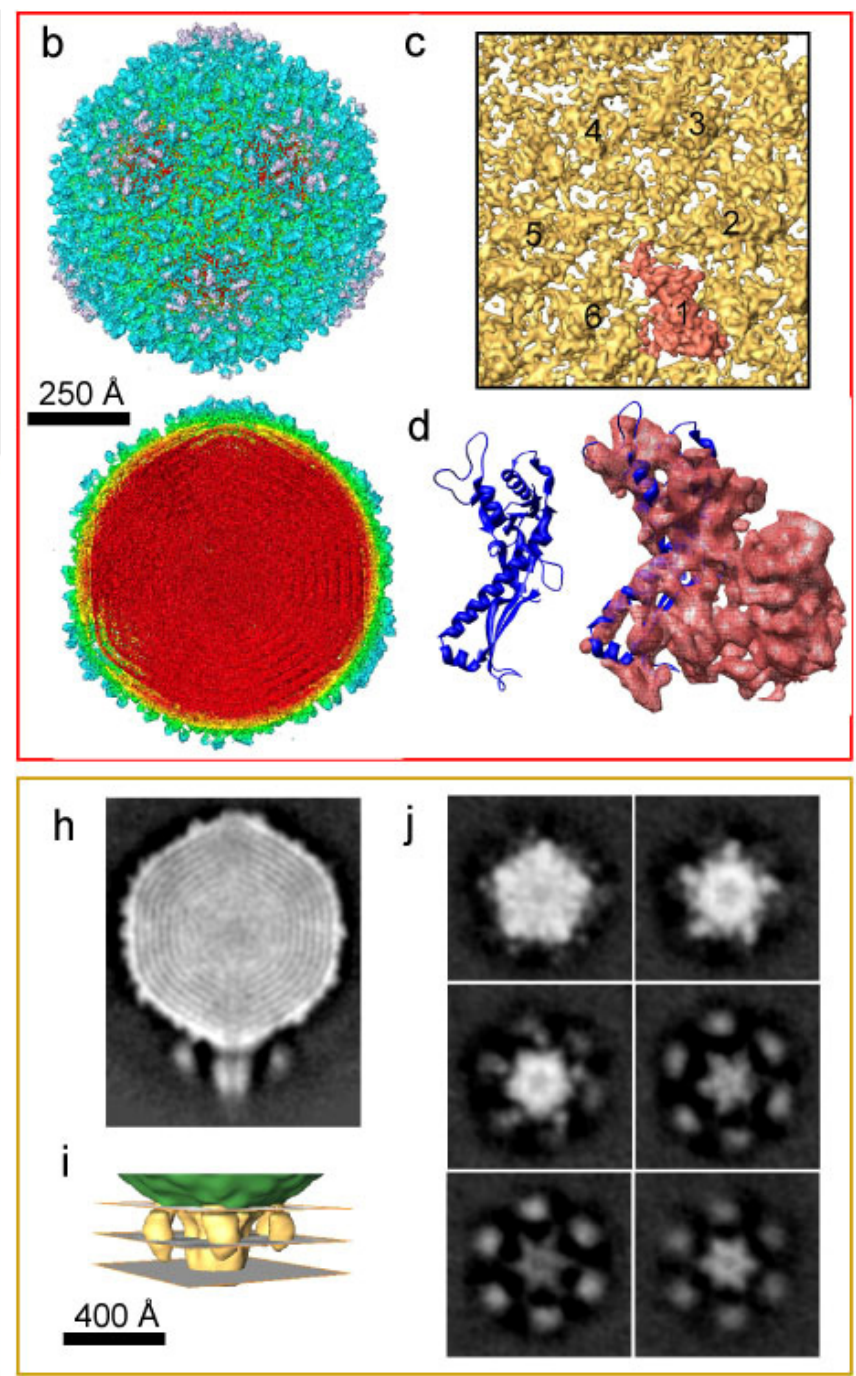

Fig. 1 Multi-scale 3D model of tropism-switching Bordetella bacteriophage by cryoEM and cryoET. (a). CryoEM images of the phage particles taken at $15.5 \mathrm{k}$ magnification. The inset shows a negative stain image of a particle with head, base plate and tail fibers. Red box: subnanometer resolution structure of the head shell (b, upper panel, and $\mathbf{c}, \mathbf{d})$ and DNA packing (b, bottom panel) by cryoEM. c, shaded surface view of a hexon. One monomer is extracted and shown in $\mathbf{d}$ in the superposition of the monomer and the HK97 head protein gp5. A long alpha helix was identified located close to the three-fold axis. Blue box: study of the interactions between the tail fiber and the base plate by cryoET. Sectional views from the top to the bottom of a representative BPP-1 3D tomogram are shown in e. The tail fibers project from the bottom of the leaflets surrounding the central cylinder of the base plate (f, g). Brown box: interactions between the base plate and the underlying penton subunits by cryoEM reconstruction without imposing symmetry. Vertical section across the base plate (h) reveals the attachment of the DNA to the penton subunits inside the shell underlying the base plate. Sequential sections $(\mathbf{i}, \mathbf{j})$ revealed the transition of the five fold symmetry of the penton subunits to the quasi six fold symmetric base plate. 